\title{
Some Properties on the Function Involving the Gamma Function
}

\author{
Bin Chen \\ Department of Mathematics and Information Science, Weinan Normal University, Weinan, China \\ Email: ccbb3344@163.com
}

Received April 25, 2010; revised May 25, 2012; accepted June 2, 2012

\begin{abstract}
We studied the monotonicity and Convexity properties of the new functions involving the gamma function, and get the general conclusion that Minc-Sathre and C. P. Chen-G. Wang's inequality are extended and refined.
\end{abstract}

Keywords: Gamma Function; Monotonicity; Convexity; Inequality

\section{Introduction}

The classical gamma function $\Gamma(x)=\int_{0}^{\infty} t^{x-1} e^{-t} \mathrm{~d} t,(x>0)$ is one of the most important functions in analysis and its applications. The logarithmic derivative of the gamma function can be expressed in terms of the series

$$
\omega(x)=\frac{\Gamma^{\prime}(x)}{\Gamma(x)}=-\gamma+\sum_{n=0}^{\infty}\left(\frac{1}{1+n}-\frac{1}{x+n}\right)
$$

$(x>0 ; \gamma=0.57721566490153286 \ldots$ is the Euler's constant), which is known in literature as psi or digamma function. We conclude from (1) by differentiation

$$
\omega^{(k)}(x)=(-1)^{k+1} k ! \sum_{n=0}^{\infty} \frac{1}{(x+n)^{k+1}},(x>0 ; k=1,2,3 \cdots)
$$

$\omega^{k}(x)$ are called polygamma functions.

H. Minc and L. Sathre [1] proved that the inequality

$$
\frac{n}{n+1}<\frac{\sqrt[n]{n !}}{\sqrt[n+1]{(n+1) !}}<1,(n=1,2, \cdots)
$$

is valid for all natural numbers $n$. The Inequality (3) can be refined and generalized as (see [2-4])

$$
\frac{n+k+1}{n+m+k+1}<\left(\sum_{i=k+1}^{n+k} i\right)^{1 / n} /\left(\sum_{i=k+1}^{n++m+k} i\right)^{1 / n+m} \leq \sqrt{\frac{n+k}{n+m+k}}
$$

where $k$ is a nonnegative integer, $n$ and $m$ are natural numbers. For $n=m=1$, the equality in (4) is valid. The Inequality (4) can be written as

$$
\begin{aligned}
\frac{n+k+1}{n+m+k+1} & <\frac{[\Gamma(n+k+1) / \Gamma(k+1)]^{1 / n}}{[\Gamma(n+m+k+1) / \Gamma(k+1)]^{1 / n+m}} \\
& \leq \sqrt{\frac{n+k}{n+m+k}}
\end{aligned}
$$

In 1985, D. Kershaw and A. Laforgia [5] showed the function $\left[\Gamma\left(1+\frac{1}{x}\right)\right]^{x}$ is strictly decreasing and $x\left[\Gamma\left(1+\frac{1}{x}\right)\right]^{x}$ strictly increasing on $(0, \infty)$, from which the Inequality (3) can be derived. In 2003, B.-N. Guo and F. Qi [2] proved that the function

$$
f(x)=\frac{[\Gamma(x+y+1) / \Gamma(y+1)]^{1 / x}}{(x+y+1)}
$$

is decreasing in $x \geq 1$ for fixed $y \geq 0$, from which the left-hand side inequality of (5) can be obtained. In the 2009 , C. P. Chen-G. Wang had obtained the extended inequality of the function above. They gave the limits of it and other results.

In this paper, our Theorem 1 considers the monotonicity and logarithmic convexity of the new function $g$ on $(0, \infty)$. This extends and generalizes B.-N. Guo and F. Qi's [2] as well as C. P. Chen and G. Wang's [6] results.

Theorem 1. Let fixed $t \geq 0$ and $s \geq 0$ be real number, then the new function

$$
g(x)=\frac{[\Gamma(x+s+1) / \Gamma(s+1)]^{1 /(x+t)}}{x+s+1}
$$

is strictly decreasing and strictly logarithmically convex on $(0, \infty)$, Moreover,

$$
\lim _{x \rightarrow 0} g(x)=e^{\omega(s+1)} /(s+1) \text { and } \lim _{x \rightarrow \infty} g(x)=e^{-1}
$$

Theorem 2. Let $k \geq 2$ be an positive integer, $s \geq 0$ be real number, then the function 


$$
h(x)=\frac{[\Gamma(x+s+1) / \Gamma(s+1)]^{1 /(x+t)}}{(x+s+1)^{1 / k}}
$$

is strictly increasing on $(0, \infty)$.

\section{Proof of the Theorems}

Proof of Theorem 1. First, we define for fixed $t \geq 0$ and $s \geq 0$,

$$
\begin{aligned}
A(x)= & (x+t)^{2} \frac{g^{\prime}(x)}{g(x)} \\
= & -\ln \frac{\Gamma(x+s+1)}{\Gamma(s+1)}+(x+t) \omega(x+s+1)-\frac{(x+t)^{2}}{x+s+1} \\
B(x)= & (x+t)^{3} \frac{\mathrm{d}^{2}[\ln g(x)]}{\mathrm{d}^{2}(x)} \\
= & 2 \ln \frac{\Gamma(x+s+1)}{\Gamma(s+1)}-2(x+t) \omega(x+s+1) \\
& +(x+t)^{2} \omega^{\prime}(x+s+1)+\frac{(x+t)^{3}}{(x+s+1)^{2}}
\end{aligned}
$$

From the differentiation of $A(x)$, we should have

$$
\begin{aligned}
& \frac{1}{(x+t)} A^{\prime}(x)=\omega^{\prime}(x+s+1)-\frac{1}{x+s+1}-\frac{1}{(x+s+1)^{2}} \\
= & \sum_{n=1}^{\infty} \frac{1}{(x+s+n)^{2}}-\sum_{n=1}^{\infty}\left[\frac{1}{x+s+n}-\frac{1}{x+s+n+1}\right] \\
& -\sum_{n=1}^{\infty}\left[\frac{s+1}{(x+s+n)^{2}}-\frac{s+1}{(x+s+n+1)^{2}}\right] \\
= & -\sum_{n=1}^{\infty}\left[\frac{s}{(x+s+n)^{2}}+\frac{1}{(x+s+n)(x+s+n+1)}\right. \\
& \left.-\frac{s+1}{(x+s+n+1)^{2}}\right] \\
= & \sum_{n=1}^{\infty} \frac{(2 s+1)(x+s+n)+s}{(x+s+n)^{2}(x+s+n+1)^{2}}<0
\end{aligned}
$$

Hence, the function $A(x)$ is strictly decreasing and $A(x)<A(0)$, for $x>0$, which yields the desired result that $g^{\prime}(x)<0$ for $x>0$.

Using the asymptotic expansion [7, p. 257]

$$
\begin{aligned}
\ln \Gamma(x)= & \left(x-\frac{1}{2}\right) \ln x-x+\ln \sqrt{2 \pi} \\
& +\frac{1}{12 x}+o\left(x^{-3}\right)(x \rightarrow \infty),
\end{aligned}
$$

and

$$
\begin{aligned}
\ln g(x)= & \frac{1}{(x+t)}[\ln \Gamma(x+s+1)-\ln \Gamma(s+1)] \\
& -\ln (x+s+1)
\end{aligned}
$$

we can conclude that $\lim _{x \rightarrow \infty} g(x)=e^{-1}$.

By L'Hospital rule, we conclude from (6) that

$$
\lim _{x \rightarrow 0} g(x)=\frac{e^{\omega(s+1)}}{(s+1)}
$$

Then from the Differentiation of $B(x)$ yields

$$
\begin{aligned}
& \frac{1}{(x+t)^{2}} B^{\prime}(x)=\omega^{\prime \prime}(x+s+1)+\frac{1}{(x+s+1)^{2}}+\frac{2(s+1)}{(x+s+1)^{3}} \\
& =-\sum_{n=1}^{\infty} \frac{2}{(x+s+n)^{3}}+\sum_{n=1}^{\infty}\left[\frac{1}{(x+s+n)^{2}}-\frac{1}{(x+s+n+1)^{2}}\right] \\
& +\sum_{n=1}^{\infty}\left[\frac{2(s+1)}{(x+s+n)^{3}}-\frac{2(s+1)}{(x+s+n+1)^{3}}\right] \\
& =\sum_{n=1}^{\infty} \frac{3(2 s+1)(x+s+n)^{2}+(6 s+1)(x+s+n)+2 s}{(x+s+n)^{3}(x+s+n+1)^{3}}>0 .
\end{aligned}
$$

Hence, the function $B(x)$ is strictly increasing and $B(x)>B(0)$ for $x>0$, which yields the desired result that $\frac{\mathrm{d}^{2}[\ln g(x)]}{\mathrm{d} x^{2}}>0$ for $x>0$.

Proof of Theorem 2. Define for $k \geq 0$ be an positive integer and $x>0$,

$$
\begin{aligned}
C(x)= & (x+t)^{2} \frac{h^{\prime}(x)}{h(x)} \\
= & -\ln \frac{\Gamma(x+s+1)}{\Gamma(s+1)}+(x+t) \omega(x+s+1) \\
& -\frac{(x+t)^{2}}{k(x+s+1)}
\end{aligned}
$$

Differentiation of $C(x)$ gives

$$
\begin{aligned}
& \frac{1}{(x+t)} C^{\prime}(x) \\
& =\omega^{\prime}(x+s+1)-\frac{1}{k(x+s+1)}-\frac{s+1}{k(x+s+1)^{2}} \\
& =\sum_{n=1}^{\infty} \frac{1}{(x+s+n)^{2}}-\frac{1}{k(x+s+1)}-\frac{s+1}{k(x+s+1)^{2}} \\
& >\int_{1}^{\infty} \frac{\mathrm{d} t}{(x+s+1)^{2}}-\frac{1}{k(x+s+1)}-\frac{s+1}{k(x+s+1)^{2}} \\
& =\frac{x}{k(x+s+1)^{2}}>0 .
\end{aligned}
$$


Hence, the function $C(x)$ is strictly increasing and $C(x)>C(0)$ for $x>0$ which yields the desired result that $h^{\prime}(x)>0$ for $x>0$.

\section{Use the Theorem}

From the proof above the following corollaries are obvious.

Corollary 1. Let fixed $t \geq 0$ and $s \geq 0$ be a real number, then for all real numbers $x>0$,

$$
e^{-1}<\frac{[\Gamma(x+s+1) / \Gamma(s+1)]^{1 /(x+t)}}{x+s+1}<\frac{e^{\omega(s+1)}}{(s+1)}
$$

Both bounds in (7) are best possible.

Corollary 2. Let fixed $t \geq 0, \alpha>0$ and $s \geq 0$ be real numbers, $k \geq 2$ be an positive integer, then for all real numbers $x>0$,

$$
\begin{aligned}
\frac{x+s+1}{x+\alpha+s+1} & <\frac{[\Gamma(x+s+1) / \Gamma(s+1)]^{1 / x+t}}{[\Gamma(x+\alpha+s+1) / \Gamma(s+1)]^{1 /(x+t+\alpha)}} \\
& <\sqrt[k]{\frac{x+s+1}{x+\alpha+s+1}}
\end{aligned}
$$

In particular, taking in (8) $t=0, k=2$, we obtain the result that Minc-Sathre and C. P. Chen-G. Wang got

$$
\begin{aligned}
\frac{x+s+1}{x+\alpha+s+1} & <\frac{[\Gamma(x+s+1) / \Gamma(s+1)]^{1 / x}}{[\Gamma(x+\alpha+s+1) / \Gamma(s+1)]^{1 /(x+\alpha)}} \\
& <\sqrt{\frac{x+s+1}{x+\alpha+s+1}}
\end{aligned}
$$

The inequality is an improvement of above, and we can extend it as the below form.

Corollary 3. Let $k \geq 2$, we have

$$
\begin{aligned}
\frac{x+s+1}{x+\alpha+s+1} & <\frac{[\Gamma(x+s+1) / \Gamma(s+1)]^{1 / x}}{[\Gamma(x+\alpha+s+1) / \Gamma(s+1)]^{1 /(x+\alpha)}} \\
& <\sqrt[k]{\frac{x+s+1}{x+\alpha+s+1}}
\end{aligned}
$$

In most particular, weobtain

Corollary 4. Let $t$ be an positive integer, we get

$$
\frac{n+1}{n+2}<\frac{\sqrt[n+t]{n !}}{\sqrt[n+t+1]{(n+1) !}}<\sqrt[k]{\frac{n+1}{n+2}}
$$

and for $k>2$,

$$
\frac{n+1}{n+2}<\frac{\sqrt[n]{n !}}{\sqrt[n+1]{(n+1) !}}<\sqrt[k]{\frac{n+1}{n+2}}
$$

Corollary 5. Let $t$ be an positive integer, we get

$$
\frac{n+1}{n+2}<\frac{\sqrt[n+t]{n !}}{\sqrt[n+t+1]{(n+1) !}}<\sqrt{\frac{n+1}{n+2}}
$$

The Inequality (13) is an improvement of (3).

\section{Acknowledgements}

Foundation item: Supported by SFC (11071194), Scientific Research Program Funded by Shaanxi Provincial Education Department (Program No 12JK0880) Shaanxi Provincial Natural Foundation (2012JM1021), Weinan Normal University Foundation (12YKS024), Key help subjects of Shaanxi Provincial Foundation. State Key Laboratory of Information Security (Institute of Software, Chinese Academy of Sciences100190) (2011NO: 01-012).

\section{REFERENCES}

[1] H. Minc and L. Sathre, "Some Inequalities Involving $(r !)^{1 / r}$," Proceedings of the Edinburgh Mathematical Society, Vol. 14, No. 65, 1964, pp. 41-46. doi:10.1017/S0013091500011214

[2] B.-N. Guo and F. Qi, "Inequalities and Monotonicity for the Ratio of Gamma Functions," Taiwanese Journal of Mathematics, Vol. 7, No. 2, 2003, pp. 239-247.

[3] F. Qi, "Inequalities and Monotonicity of Sequences Involving $\sqrt[n]{(n+k) ! / k !}$," Soochow Journal of Mathematics, Vol. 29, No. 4, 2003, pp. 353-361.

[4] F. Qi and Q.-M. Luo, "Generalization of H. Minc and J. Sathre's Inequality," Tamkang Journal of Mathematics, Vol. 31, No. 2, 2000, pp. 145-148.

[5] D. Kershaw and A. Laforgia, "Monotonicity Results for the Gamma Function," Atti Accad. Sci. Torino Cl. Sci. Fis. Mat. Natur., Vol. 119, 1985, pp. 127-133.

[6] C.-P. Chen and G. Wang, "Monotonicity and Logarithmic Convexity Properties for the Gamma Function," Scientia, Vol. 5, No. 1, 2009, pp. 51-54.

[7] M. Abramowitz and I. A. Stegun (Eds.), "Handbook of Mathematical Functions with Formulas, Graphs, and Mathematical Tables, National Bureau of Standards," Applied Mathematics Series, 4th Printing, Washington, Vol. 55, 1965. 